\title{
Evaluación del potencial eólico y predicción de la velocidad de viento con Minería de Datos
}

\author{
Fecha de recepción: 2020-06-05 • Fecha de aceptación:2020-07-15 • Fecha de publicación: 2020-10-10
}

\author{
Carlos Luis Vargas Guevara \\ Universidad Técnica de Ambato, Ecuador \\ cvargas0028@uta.edu.ec \\ https://orcid.org/0000-0003-2050-2342
}

\begin{abstract}
El flujo del viento se ha visto afectado por factores como la rugosidad y la topografía del terreno que producen perturbaciones en su movimiento. La presencia de colinas puede generar, por un lado, aumento de velocidad en el viento hasta llegar a la cima, pero, a su vez, en la parte inferior aumenta la turbulencia produciendo efectos de recirculación. Este comportamiento no permite conocer en detalle el desarrollo de los perfiles de velocidad y la energía cinética turbulenta del viento. En este sentido, por el gran interés en el estudio del flujo de aire en terrenos complejos, el presente proyecto tiene como objetivo la evaluación del recurso eólico en terrenos complejos, específicamente en un túnel de viento natural ubicado entre el nevado Chimborazo y Carihuairazo del Ecuador, además de la predicción de la velocidad para un nuevo horizonte de tiempo.
\end{abstract}

PALABRAS CLAVE: minería de datos, energía, generación, predicción, velocidad, viento.

\section{RESUMEN}

The flow of the wind has been affected by factors such as roughness and topography of the terrain, which produce disturbances in its movement. The presence of hills can generate, on the one hand, 
an increase in wind speed until reaching the top, but in turn, in the lower part it increases turbulence producing recirculation effects. This behavior does not allow to know in detail the development of the velocity profiles and the turbulent kinetic energy of the wind. In this sense, due to the great interest in the study of air flow in complex terrains, the present project consists of the evaluation of the wind resource in complex lands, specifically in a natural wind tunnel located between the snow-covered Chimborazo and Carihuairazo of Ecuador, and the prediction of the wind speed for a new time horizon. 


\section{Introducción}

La energía es un recurso que se ha convertido en uno de los pilares fundamentales de las economías actuales por su facilidad para distribuirla (Torrijos Moreno, J., 2019). La predicción de energía renovable facilita que nuevas fuentes de energía eléctrica alcancen un mayor interés en las redes eléctricas actuales y es uno de los soportes para las redes eléctricas del futuro o más conocidas como "Smart grids". Una vez que se consigue la independencia energética y la robustez a la producción de energía eléctrica proveniente de combustibles fósiles se minimizan las fluctuaciones de los precios por diversos motivos, entre ellos el impacto ambiental causado por la gestión de residuos (Medina \& Juan, 2012).

Un factor común que se presenta en la energía solar y eólica es la aleatoriedad. Por lo impredecible del potencial energético extraíble de la atmósfera, la mayoría de los enfoques de predicción se basan en modelos físicos que emplean simulaciones numéricas del tiempo. Asimismo, la variabilidad es el factor por el que las energías renovables siguen teniendo una escasa penetración, una técnica para estimar la incertidumbre de la variable predicha son los métodos de predicción denominados probabilistas (Medina \& Juan, 2012).

La energía eólica ha tenido un desarrollo importante en la generación de electricidad. En en el año 2012 se registró una potencia de $282,59 \mathrm{GW}$ instalados en el mundo, esta se encuentra distribuida de la siguiente manera: $109.58 \mathrm{GW}$ para Europa, $97.57 \mathrm{GW}$ instalados en Asia, 67.58 GW en América del Norte, 3.22 GW en la región del Pacífico, 1.14 GW en África y medio oriente y finalmente 3.51GW en América Latina y el Caribe (Cortes - Pérez, Sierra-Vargas, \& Arango Gómez, 2016).

Un tema de interés son las predicciones probabilísticas, el índice de riesgo y las proyecciones espacio-tiempo en rangos muy cortos, cortos, medianos y largo plazo. De esta manera se desarrollan cuatro aspectos en los que se incluyen: la distribución predictiva, los parámetros de localización, escala y la evaluación (Cortes - Pérez, Sierra-Vargas, \& Arango - Gómez, 2016).

La contaminación ambiental y el consumo de combustibles fósiles para obtener energía eléctrica se incrementan anualmente. La mitigación de este problema exige la búsqueda de nuevas fuentes de energía alternativas, amigables con el medio ambiente y que contribuyan a la conservación del planeta, de esta manera, una de las soluciones más relevantes asociadas a la generación de energía limpia es la explotación del recurso eólico (Lawan, Abidin, Lawan, Bichi, \& Abba, 2017). No obstante, el flujo del viento se ve afectado por factores como la rugosidad y la topografía del terreno, es así que se deben implementar técnicas y metodologías de aprovechamiento del potencial eólico que evalúen y pronostiquen la velocidad de este recurso. La presencia de colinas, por ejemplo, puede ser por un lado beneficiosa, ya que la velocidad del viento aumenta al llegar a la cima, pero, por otro lado, pendiente abajo, la turbulencia se ve incrementada, produciendo efectos de recirculación. Este comportamiento no permite conocer con detalle el desarrollo de los perfiles de velocidad y la energía cinética turbulenta del viento, en este sentido, existe en la actualidad un gran interés sobre el estudio del flujo de aire en terrenos complejos. Es así como, en el presente artículo, se abordará la predicción de energía a través de técnicas de Minería de 


\section{Datos.}

En el presente artículo se describe la evaluación del recurso eólico en terreno complejo, específicamente en un túnel natural de viento ubicado entre los nevados Chimborazo y Carihuayrazo en la cordillera occidental de los Andes del Ecuador, además de la predicción de generación de energía eléctrica eólica en un horizonte de 24 horas. El aporte científico de la presente propuesta es la evaluación del potencial eólico del sector en estudio y la predicción de la velocidad del viento empleando técnicas de minería de datos.

\section{Análisis y revisión de métodos de predicción}

En los últimos años las energías renovables han presentado una gran penetración en los sistemas eléctricos de diferentes países del mundo, por lo que se han desarrollado varios estudios en cuanto al monitoreo y predicción de la generación eléctrica. Las estructuras complejas de la superficie de la tierra, tales como colinas, cambian profundamente el flujo de viento, afectando la velocidad y dirección. En la cima de las colinas la velocidad de viento puede ser incrementada en forma significativa, debido a la compresión de las líneas de flujo, esto se conoce como el efecto colina. Estos incrementos de la velocidad de viento son una de las razones por las que las turbinas eólicas se instalan en la cima. La presencia de valles entre colinas produce el efecto túnel, donde la dirección del viento se desvía considerablemente del flujo de aire no perturbado (Medina \& Juan, 2012).

Para el estudio del flujo de aire sobre colinas se han desarrollado una variedad de modelos y técnicas numéricas, estas interpretan y resuelven las ecuaciones fundamentales del flujo de fluidos como son la conservación de la masa, la conservación de la energía y la cantidad de movimiento para flujo viscoso representadas en un análisis estadístico multivariante a través de técnicas como la correlación.

Se propone un modelo de predicción estadístico/CFD basado en Random Forests, ya que este utiliza la distribución de Weibull para el análisis de los datos medidos, el modelo es capaz de reproducir la variación espacial de la velocidad de viento sobre terreno complejo, luego es validado en un túnel de viento para mejorar la precisión para la predicción.

\section{Metodología}

Como métodos estadísticos que poseen una caja negra están los análisis de series temporales. Una ventaja de estos métodos es la capacidad de modelar en una sola etapa la predicción meteoenergética que se produce en la etapa de adaptación/entrenamiento.

A su vez, están los métodos conocidos como "predicción numérica del tiempo - NWP". Estos modelan la dinámica y la física de la atmósfera, entre los más usados resaltan los globales y los de meso escala.

Una tercera técnica son los denominados MOS (Model Output Statistics), que se basan en tomar la predicción de un NWP más un conjunto de observaciones pasadas para emitir una nueva 
predicción. Una consideración especial es que en un horizonte de predicción comprendido de una a seis horas, los métodos estadísticos suelen ser relativamente mejores (Medina \& Juan, 2012).

Por otra parte, otros utilizados son los métodos de predicción probabilista, estos presentan un nivel de formalismo relacionado con el estudio de la incertidumbre. Existen dos de este tipo: los denominados "ensemble", cuyo significado es predicción por conjuntos y los que probabilizan una predicción puntual (Bayram, Murat, Tahir, \& Ziyaddim, 2018). Una de las ventajas de este método es la capacidad de determinar la predictibilidad de cada región utilizando condiciones actuales. Una variante menos costosa que ensemble es la "Poor man's ensemble", que toma predicciones en distintos momentos ya pasados, o empleando modelos ya existentes (Medina \& Juan, 2012).

El conjunto de datos empleado para el presente trabajo ha sido proporcionado por el grupo de investigación Reward de la Universidad Técnica de Ambato. Los datos medidos corresponden a mediciones realizadas en una torre de 20 metros de altura, esta torre se encuentra en la comunidad de Mechahuasca, ubicada entre las provincias de Bolívar y Chimborazo.

Los datos adquiridos tienen una frecuencia de 10 minutos y corresponden a mediciones realizadas en el año 2018 entre los meses de enero a mayo, sumando un total de 19657 datos, en la Tabla 1 se visualizan las magnitudes adquiridas y el formato de cada una.

Tabla 1.

Magnitudes adquiridas

\begin{tabular}{cc}
\hline MAGNITUD & FORMATO \\
\hline Fecha & $\mathrm{Dd} / \mathrm{mm} / \mathrm{aa}$ \\
\hline Tiempo & $\mathrm{Hh} / \mathrm{mm} / \mathrm{ss}$ \\
\hline Datos & Numérica ascendente \\
\hline Velocidad del Viento & $\mathrm{m} / \mathrm{s}$ \\
\hline Temperatura & ${ }^{\circ} \mathrm{C}$ \\
\hline Presión Atmosférica & Bares \\
\hline Radiación solar & $\mathrm{w} / \mathrm{m}^{2}$ \\
\hline Dirección del viento & $\mathrm{Grados}$ \\
\hline Humedad & $\mathrm{HR}$ \\
\hline
\end{tabular}

Fuente: elaboración propia

El conjunto de datos permite obtener en si una matriz de datos $\mathbf{X}_{(\mathbf{n x p})}$, en donde $(\mathbf{n})$ es el número de individuos de la matriz de datos, mientras que (p) representa al número de variables que se relacionan con dichos individuos. En la presente investigación se va a trabajar con una matriz de datos de dimensiones 19657 individuos por 6 variables.

\section{Análisis estadístico multivariante}

A partir de la matriz de datos obtenida se pueden realizar varios análisis de datos. Uno de los más empleados para describir el comportamiento de las variables es el análisis estadístico 
multivariante. De esta manera se analiza la relación de las variables con cada uno de los individuos de la matriz de datos, en este sentido se aplican las diferentes medidas estadísticas (Bektas, Kücükdeniz , \& Özcan, 2017).

La desviación estándar es una medida que se usa para cuantificar la variación o dispersión de un conjunto de datos numéricos (Borovsky, 2019). En (1) se presenta la ecuación que permite la desviación estándar con la que se realizan las estimaciones para minimizar los errores aleatorios.

$$
\sigma=\sqrt{\frac{1}{N} \sum_{i=1}^{N}\left(x_{i}-\mu\right)^{2}}
$$

En Python obtener la desviación estándar es sencillo ya que se dispone de la función (std). En la Tabla 2 se muestran los valores de desviación estándar alcanzados en el presente análisis.

Tabla 2.

Valores de Desviación Estándar

\begin{tabular}{ll}
\hline Variable & $\begin{array}{l}\text { Valor de Desviación } \\
\text { Estándar }\end{array}$ \\
\hline VELOCIDAD_VIENTO & 4.262831 \\
\hline TEMPERATURA & 2.226710 \\
\hline PRESION_ & 1.181077 \\
ATMOSFERICA & \\
\hline RADIACION_SOLAR & 278.814786 \\
\hline DIRECCION_VIENTO & 82.944563 \\
\hline HUMEDAD & 22.524698 \\
\hline
\end{tabular}

Fuente: elaboración propia

Por otra parte, la varianza de una variable aleatoria es una medida de dispersión definida como la esperanza del cuadrado de la desviación de dicha variable respecto a su media, la fórmula que la representa se muestra en (2).

$$
\sigma^{2}=\frac{1}{n} \sum_{i=1}^{n}\left(x_{i}-\bar{X}\right)^{2}
$$


Mientas que los datos de las varianzas analizadas se aprecian en la Figura 1.

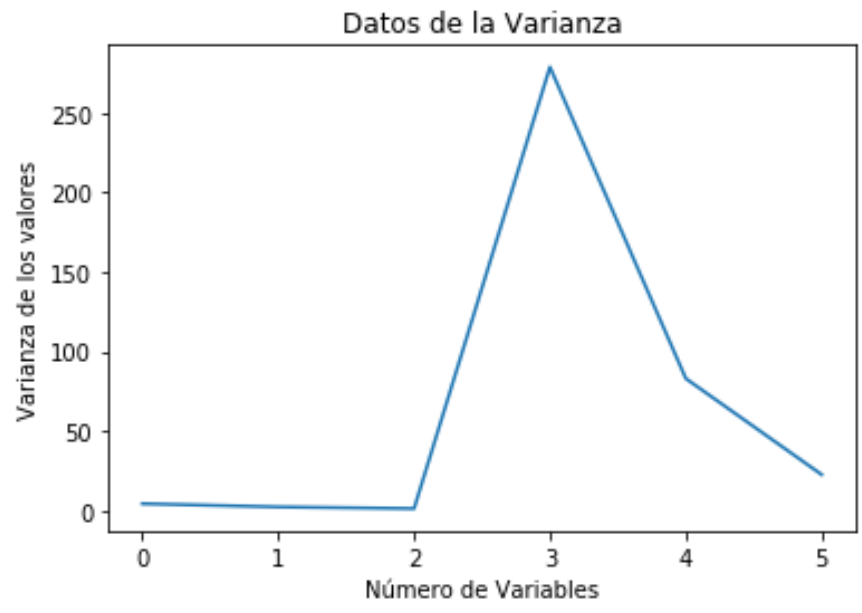

Figura 1. Datos de la Varianza

Fuente: elaboración propia

La medida de tendencia central más conocida y utilizada es la media aritmética o promedio aritmético. En la Tabla 3 se presentan los valores promedio de las variables analizadas.

Tabla 3.

Valores promedio de las Variables

\begin{tabular}{ll}
\hline Variable & Valor Promedio \\
\hline VELOCIDAD_VIENTO & 7.969444 \\
\hline TEMPERATURA & 4.28506 \\
\hline PRESION_ATMOSFERICA & 611.996 \\
\hline RADIACION_SOLAR & 180.163 \\
\hline DIRECCION_VIENTO & 116.152 \\
\hline HUMEDAD & 65.9564 \\
\hline
\end{tabular}

Fuente: elaboración propia

Otra medida de tendencia central es la mediana, esta corresponde al valor de la variable que ocupa la posición central, cuando los datos se disponen en orden de magnitud (Piazzi, 2017). En la Tabla 4 se evidencian los valores asociados a los valores de mediana. 
Tabla 4.

Valores de mediana de las Variables

\begin{tabular}{ll}
\hline Variable & Valor Mediana \\
\hline VELOCIDAD_VIENTO & 7.9 \\
\hline TEMPERATURA & 3.7 \\
\hline PRESION_ & 612.0 \\
ATMOSFERICA & \\
\hline RADIACION_SOLAR & 2.3 \\
\hline DIRECCION_VIENTO & 79.0 \\
\hline HUMEDAD & 63.8 \\
\hline
\end{tabular}

Fuente: elaboración propia

\section{Análisis Gráfico}

El análisis gráfico es una forma sencilla de representar una situación, problema, esquema, sistema o en general para representar las características de un conjunto de datos (Sun, 2017). De esta manera se proporciona al lector la mayor cantidad de información con solo un vistazo. En datos multivariantes es difícil transmitir la información y no resulta sencillo su elaboración. En este sentido, el software de análisis de datos Python integra librerías como matplotlib que facilitan la elaboración de estos. En la Figura 2 se muestra la distribución de los datos en forma gráfica.
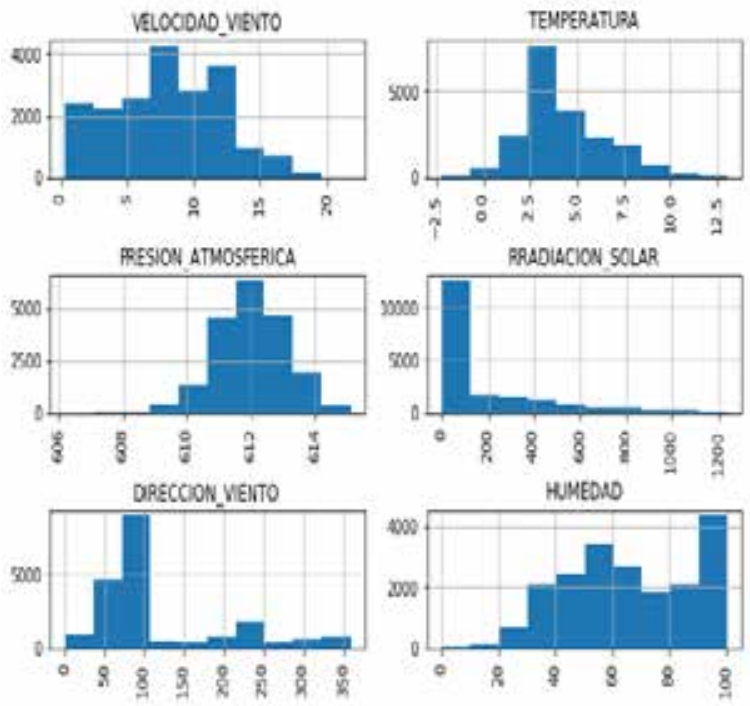

Figura 2. Distribución de Datos por Variable

Fuente: elaboración propia.

Parte del análisis gráfico asociado a los valores de variables es la detección de valores extraños o atípicos que se pueden realizar en el proceso de medición (Narayana, 2017). Generalmente los datos atípicos se encuentran entre 1-3\% en experimentos no controlados y alrededor de un $5 \%$ en experimentos no controlados, una forma de detectar valores atípicos es gracias al empleo de 
los diagramas de caja. La caja central indica el rango en el que se concentra el $50 \%$ central de los datos. La línea central representa a la mediana, mientras que los extremos son los "bigotes" y delimitan el $95 \%$ de los datos. Los datos atípicos suelen estar representados fuera de los bigotes, como se puede apreciar en la Figura 3.

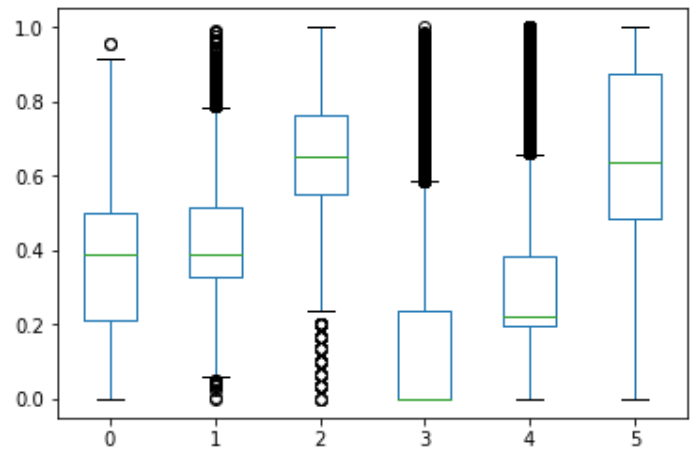

Figura 3. Diagrama de caja de las variables analizadas

Fuente: elaboración propia

La matriz de correlación es una matriz conformada por (n) filas y por (n) columnas. Además, es una matriz simétrica; es decir, que los valores de los elementos $\mathbf{a}_{[\mathrm{i}, \mathrm{j}]}$ de la matriz son el mismo valor en los elementos $\mathbf{a}_{[\mathrm{iij}}$ de la matriz. Esta nos explica cómo se encuentran relacionadas cada una de las variables con otra variable. Su diagonal siempre contendrá el valor de 1 , si tiene un valor 0 nos indicará que no tiene ninguna relación con esa variable, por lo menos no lineal; es decir, puede que tenga una relación cuadrática o de otro grado (Goh, Lee, Chua, Goh, \& Teo, 2016).

Mientras que cuando la correlación es positiva, nos indica que su proyección de la regresión lineal va a tender a crecer juntamente con la contra variable, y cuando es negativa nos muestra que su proyección de la regresión lineal va a tender a decrecer juntamente con la contra variable. En la Figura 4 se puede ver la matriz de correlaciones.

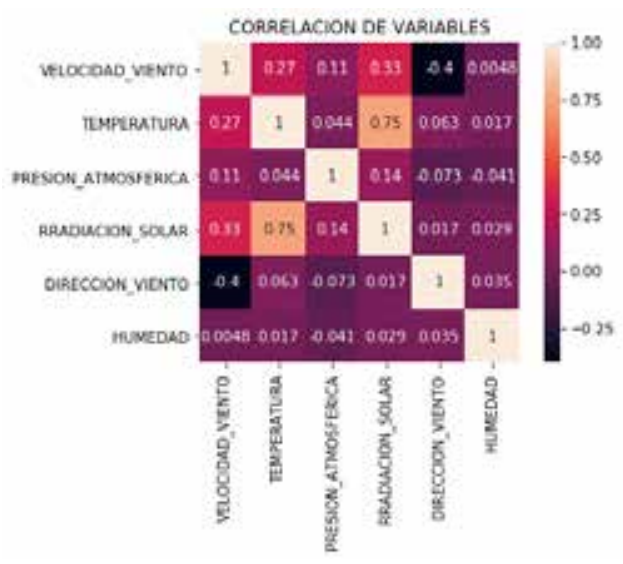

Figura 4. Matriz de correlación de las variables

Fuente: elaboración propia 


\section{Técnicas de Predicción}

Para el estudio del potencial de energía eólico las variables que se consideran más importantes son la velocidad y dirección del viento. Muchos expertos mencionan que un error de medición del $1 \%$ puede ocasionar una desviación alrededor del $2 \%$ en la producción de energía (Fischer, Montuelle, Mougeot, \& Picard, 2017). Además, ningún modelo matemático, ya sea físico o numérico, proporciona una solución perfecta y definitiva (Chen \& Pang, 2019). En los últimos años se han empleado redes neuronales artificiales (ANN) para predicciones de velocidad y potencia del viento. Entre las principales ventajas de las redes neuronales destacan: tolerancia a fallos, topología simple, tiempo de computación rápido y capacidad de estimular funciones no lineales (Mercado, García Fernández, \& Acebey, 2016).

La tarea de predicción también puede formularse como un problema de regresión. En este sentido se presentan diferentes técnicas, tales como: regresión lineal y regresión de vectores de soporte. En una lineal se espera que el valor de predicción $f(x)$ sea una combinación lineal de las variables de entrada: $f(w, x)=w 0+w 1 x 1+\cdots+w N x N$. El objetivo es encontrar los coeficientes $w=(w 1$, ....., wN) T que minimiza la suma residual de los cuadrados entre las etiquetas observadas y (y) las respuestas predichas por la aproximación lineal (Chen, Zeng, Zhou, Du, \& Lu, 2018). Por otro lado, la regresión de vectores de soporte es una de las técnicas más avanzadas para las tareas de predicción, esta se basa en las máquinas de vectores de soporte (SVM) propuestas por Vapnik en 1995. La media absoluta de error es 0.906 y la media al error cuadrado es 0.934 del modelo de predicción de regresión lineal para las cinco variables objetivo. Los resultados muestran que la técnica SVR incluso logra mejores resultados que la regresión lineal si uno toma solo las medidas absolutas como características y selecciona el número de pasos pasados con cuidado (SierraVargas \& Arango-Gómez, 2016).

Una predicción precisa de la energía eólica a corto y largo plazo es importante para un equilibrio seguro y sostenible de la red eléctrica. El comportamiento de una regresión logística polinomial con solo la velocidad del viento como covariable es, al mismo tiempo, simple y efectivo para la predicción de la potencia. Es interesante notar que los métodos agnósticos son en este caso muy apropiados para la predicción y muestran resultados prometedores con la mejor estabilidad (Parreño \& Gomez , 2004).

La generación de energía eléctrica a través de aerogeneradores es una de las alternativas de generación prácticamente inagotable (Optis \& Perr-Sauer, 2019). Se considera una fuente de energía limpia, pero aún necesita mucha investigación para el desarrollo de la ciencia y las tecnologías que garanticen la uniformidad en la generación, proporcionando una mayor participación de esta fuente en la matriz energética, ya que el viento presenta variaciones abruptas en la velocidad (Feng, Cui, Hodge, \& Zhang, 2017). En los sistemas eléctricos basados en el viento es esencial predecir al menos con un día de antelación los valores futuros del comportamiento del viento, a fin de evaluar la disponibilidad de energía para el próximo período. Este documento desarrolla modelos de predicción ultracortos, cortos, medianos y a largo plazo de la velocidad del viento, basados en técnicas de inteligencia computacional, utilizando modelos de redes neuronales artificiales, promedios móviles integrados autoregresivos (ARIMA) y modelos 
híbridos que incluyen pronósticos con wavelets (Palomares Losada, 2002, Ali, Hassan, Ali, \& Kumar, 2017).

Actualmente los procesos de predicción están integrando nuevas técnicas que los convierten en sistemas inteligentes. El aprendizaje supervisado está compuesto por dos fases o etapas, la primera llamada entrenamiento, donde se observa las características más relevantes de un evento que actúan como datos de entrada y la segunda conocida como prueba, en esta se compara las características con nuevos datos de prueba y se obtiene al final de estas dos etapas: un modelo entrenado y probado que intenta explicar los datos de entrada. Prieto Emhart (2018) presenta la aplicación del algoritmo propuesto por Huang que se denomina "Extreme Learning Machine". Este algoritmo está atrayendo la atención de miles de investigadores como una técnica de inteligencia artificial que resuelve muchos de los problemas a los que se enfrentan otras técnicas, por ejemplo, su lenta velocidad de aprendizaje (Pascual, y otros, 2010).

\section{Resultados}

El análisis de datos permitió identificar que la mayor cantidad de datos atípicos se encuentra en los valores de la radiación solar, esto revela que el instrumento de medición está mal configurado. Por otra parte, en la Figura 5 se muestra el diagrama de valores de dirección del viento, de esta manera se evidencia que la mayor concentración de datos se encuentra entre 45 grados tomados desde el norte geográfico.

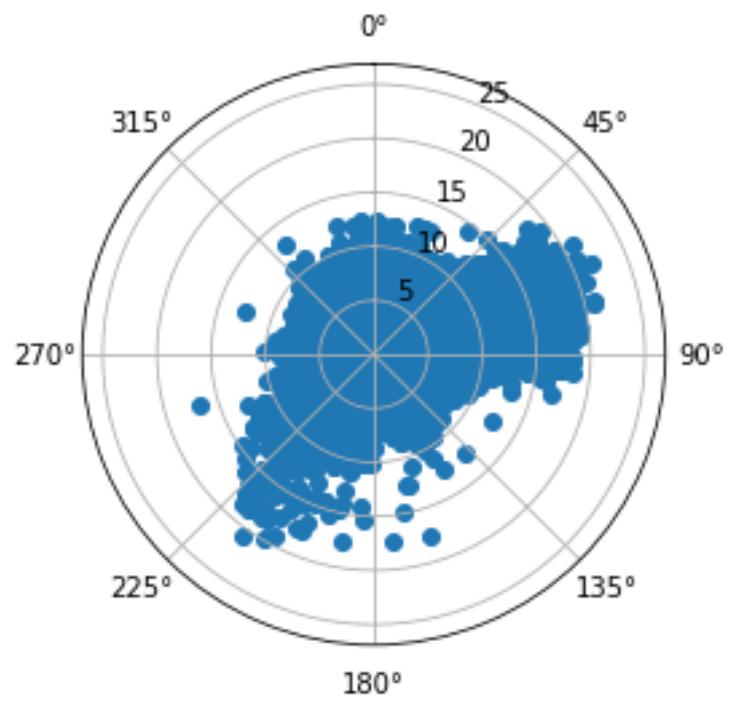

Figura 5. Diagrama de Viento

Fuente: elaboración propia

Una de las técnicas analizadas para la predicción de valores futuro es el regresor. Sin embargo, el uso de equipos informáticos permite asertividad de los métodos de predicción para que sea más alta. Esta rama de estudios es conocida como inteligencia computacional. Para el desarrollo del estudio se ha realizado una comparación entre 3 técnicas de inteligencia computacional (árboles 
de decisión, máquina de soporte vectorial y red neuronal artificial) que son las más conocidas y empleadas para estudios de predicción. La estructura de la red neuronal se seleccionará mediante un método exhaustivo en el que se probarán varios números de neuronas. La mejor estructura seleccionada será con la que el modelo presente el menor error cuadrático medio en el conjunto de test.

En la Figura 6 se puede ver la velocidad del viento estimada por un árbol de decisión. En Python se ha empleado el comando clf_DT=tree.DecisionTreeRegressor() que permite estimar los valores a futuro a través de una regresión.

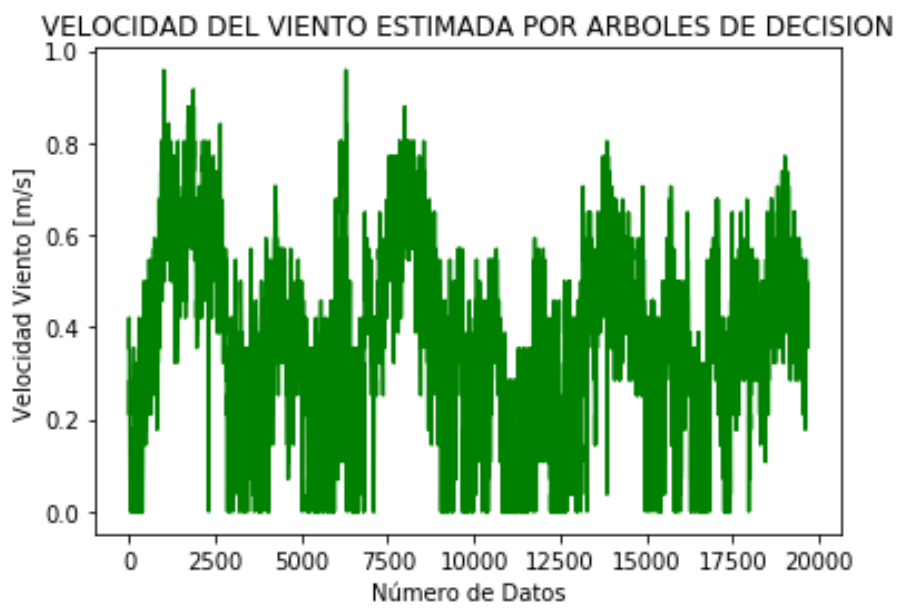

Figura 6. Valores de Velocidad de Viento con árbol de decisión

Fuente: elaboración propia

Asimismo, en la Figura 7 se evidencian los valores que pueden aparecer con el mínimo error al emplear árboles de decisión (DT). El intervalo de confianza es calculado como el menor rango de potencia que acumule el $95 \%$ de probabilidad de ocurrencia en el histograma de salida. Además, se ha realizado una comparación con una predicción simple que consiste en calcular la potencia utilizando como entrada al modelo directamente la predicción de velocidad de viento. El método es computacionalmente eficiente, se requieren menos de 10 segundos para obtener una predicción de potencia para las próximas 36 horas.

De esta manera el valor de $3 \mathrm{~m} / \mathrm{s}$ tiene mayor probabilidad de aparición con más del 1.4 de probabilidad. 


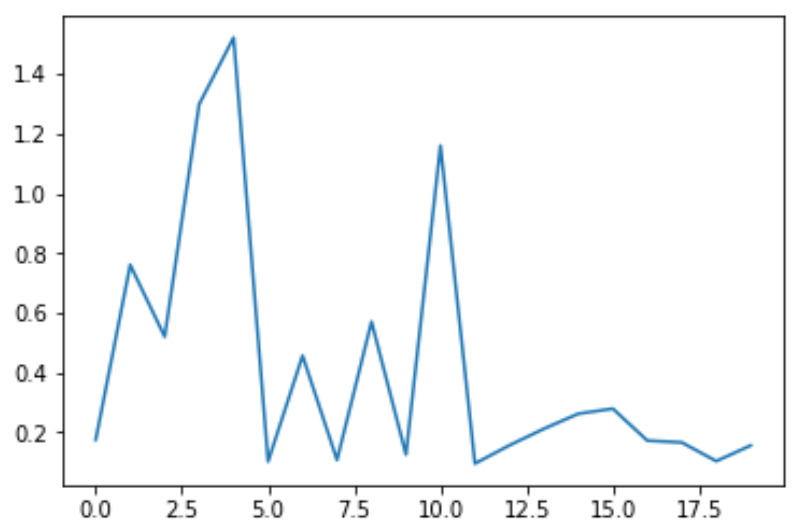

Figura 7. Valores de velocidad del viento estimados por DT

Fuente: elaboración propia

Ahora bien, el empleo de una máquina de soporte vectorial permite reducir el error de los valores estimados. No obstante, una técnica con mayor robustez es la red neuronal artificial. Esta técnica de inteligencia computacional permite dividir al total de los datos en dos grupos conocidos como prueba y entrenamiento o en términos anglosajones training and testing. El modelo que mostró mejores resultados es la red neuronal MLP con una mejora de un $62 \%$ en el error cuadrático medio con respecto al modelo de referencia, superando ampliamente al modelo ARX, que mostró un progreso del $25 \%$ y al modelo FIR, que mostró un $10 \%$. Para la calibración de estos modelos se asume conocida con exactitud la velocidad del viento en el futuro.

En la Figura 8 se reflejan los gráficos del entrenamiento de la red neuronal empleado en el presente trabajo.

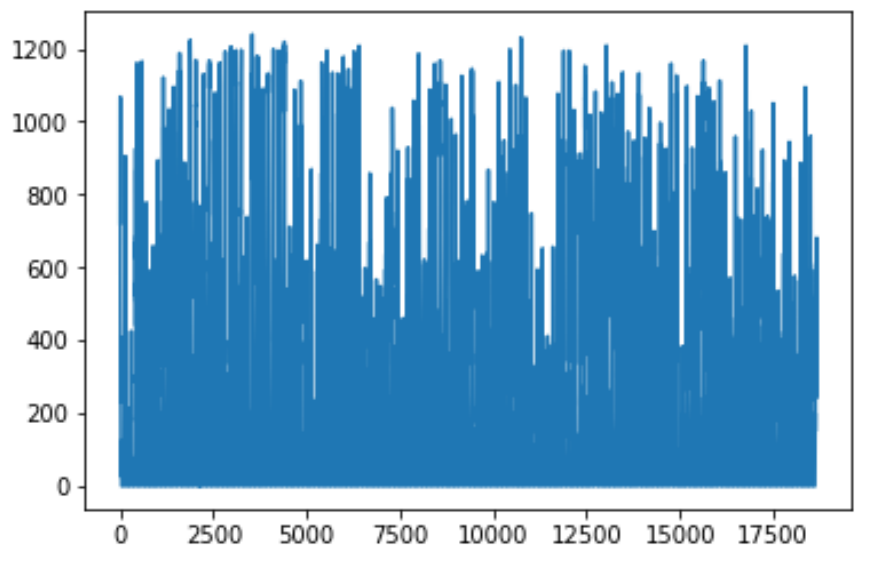

Figura 8. Datos de entrenamiento de la Red Neuronal

Fuente: elaboración propia

Asimismo, en la Figura 9 se presentan los datos del testing o prueba de la red neuronal. 


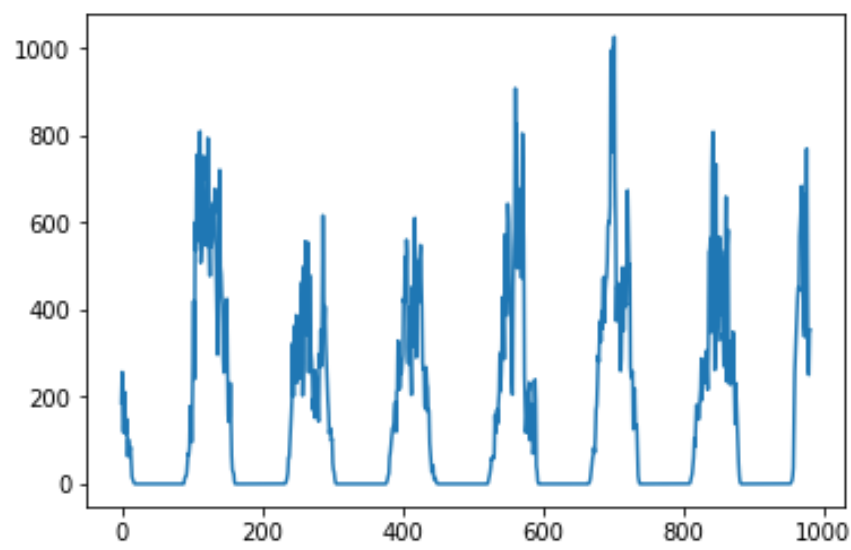

Figura 9. Datos de prueba para la Red Neuronal

Fuente: elaboración propia

Finalmente, en la Figura 10 se muestran los datos estimados con mínimo error que pueden aparecer al emplear una red neuronal.

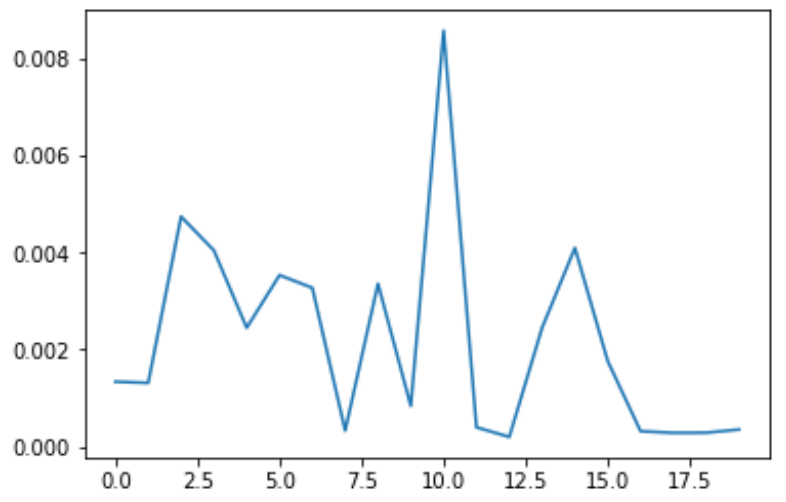

Figura 10. Datos estimados con mínimo error por la Red Neuronal

Fuente: elaboración propia

De esta manera, una vez realizadas las diferentes simulaciones, se elige a la red neuronal como la mejor técnica de inteligencia computacional, ya que presenta un error del $0.002 \%$. Lo que indica que los datos de velocidad de viento con mayor probabilidad de ocurrencia se encuentran alrededor de los $10 \mathrm{~m} / \mathrm{s}$. Además, la presencia de datos con velocidades mayores a $15 \mathrm{~m} / \mathrm{s}$ será muy escaza.

\section{Conclusiones}

La medida de la incertidumbre fue abordada en términos del valor del MAE para el periodo de calibración, se plantea la posibilidad de mejoras significativas, en la medida que se incorpore tanto asimilación de datos como corridas de ensembles. 
El tratamiento de la incertidumbre amerita mayor profundidad de análisis. Un abordaje del tipo dinámico puede ser planteado en términos de considerar la incertidumbre como una variable a pronosticar. En este caso el modelo puede ser corrido con perturbaciones en las condiciones iniciales generando pronósticos de potencia.

El análisis multivariante permite conocer la relación que existe entre las variables y describir el comportamiento actual de los datos para estudios futuros. Mientras que mediante el análisis gráfico el lector se puede determinar los valores atípicos que se encuentran en el caso de estudio, de esta manera se recomienda estudiarlos por separado.

Las técnicas de inteligencia computacional han alcanzado una mayor acogida en el proceso de determinar valores o comportamientos a futuro de las variables estudias. Todo esto es gracias al desarrollo de los recursos computacionales hardware y software.

Las redes neuronales artificiales son técnicas que presentan mayor robustez en la estimación del comportamiento de datos ya que sus niveles de error son mínimos. 


\section{Referencias}

Ali, M. E., Hassan, M. Z., Ali, M. S., \& Kumar, J. (2017). Prediction of Wind Speed Using Real Data: An Analysis of Statistical Machine Learning Techniques . Asia-Pasific World Congr. Comput. Sci. Eng. (APWC CSE), 259-264.

Bayram, K., Murat, D., Tahir, G. M., \& Ziyaddim, R. (2018). ESTIMATING WIND ENERGY POTENTIAL WITH PREDICTING BURR LSM PARAMETERS: A DIFFERENT APPROACH. Journal of Engineering \& Natural Sciences , 389-404.

Bektas, Z., Kücükdeniz , T., \& Özcan, T. (2017). A Comparison of Support Vector Regression and Multivariable Grey Model for Short-Term Wind Speed Forecasting. Turkish Journal of Forecasting,, 46-53.

Borovsky, J. E. (2019). Compacting the description of a time-dependent multivariable system and its multivariable driver by reducing the state vectors to aggregate scalars: the Earth's solar-wind-driven magnetosphere. . Nonlinear Processes in Geophysics, 429-443.

Chen, C., \& Pang, Y. (2019). Exploring Machine Learning Techniques for Smart Drainage System. 2019 IEEE Fifth International Conference on Big Data Computing Service and Applications (BigDataService), 63-70.

Chen, J., Zeng, G.-Q., Zhou, W., Du, W., \& Lu, K.-D. (2018). Wind speed forecasting using nonlinear-learning ensemble of deep learning time series prediction and extremal optimization. Energy Conversion and Management, 681-695.

Cortes - Pérez, D. M., Sierra-Vargas, F. E., \& Arango - Gómez, J. E. (2016). Evaluación, predicción y modelación del potencial eólico Assessment forescasting and modelling of wind Potencial. Ingeniería Mecánica, 167175.

Feng , C., Cui, M., Hodge, B.-M., \& Zhang, J. (2017). A data-driven multi-model methodology with deep feature selection for short-term wind forecasting. Applied Energy, 1245-1257.

Fischer, A., Montuelle, M., Mougeot, M., \& Picard, D. (2017). Statistical learning for wind power: A modelling and stability study towards forecasting. Wind Energy, 20(12), 2037-2047.

Goh, H. H., Lee, S. W., Chua, Q. S., Goh, K. C., \& Teo, K. K. (2016). Wind energy assessment considering wind speed correlation in Malaysia. Renewable and Sustainable Energy Reviews, 1389-1400.

Lawan, S. M., Abidin, W. A., Lawan, A. M., Bichi, S. L., \& Abba, I. (2017). The potential of topographical feedforward neural network (T-FFNN) technique in monthly wind speed and direction prediction. Informatics Sustain Soc. Through Digit Innov. ICEEI, 1-6.

Medina, N., \& Juan, R. (3 de Diciembre de 2012). Predicción con incertidumbre en meterolog\{ia energética. 
Aplicación en predicción eólica. Gran Canaria: ULPGC Universidad las Palmas de Gran Canaria. https:// accedacris.ulpgc.es/bitstream/10553/9120/6/tfm_rnebot.pdf

Mercado, F. R., García Fernández, W., \& Acebey, J. H. (2016). Sistema de inteligencia artificial para la predicción temprana de heladas meteorológicas. Artifitial intelligence system for early prediction of weather frost, 483-495.

Narayana, M. S. (2017). Adaptive linear prediction for optimal control of wind turbines. Renewable Energy , 895906.

Optis, M., \& Perr-Sauer, J. (2019). The importance of atmospheric turbulence and stability in machine-learning models of wind farm power production. Renewable and Sustainable Energy Reviews, 27-41.

Palomares Losada, A. M. (2002). Caracterización del régimen de vientos y desarrollo de un modelo de predicción eólica a escala local en el estrecho de Gibraltar. Madrid: Universidad Complutense de Madrid.

Parreño, J., \& Gomez , A. (2004). Pronostico de la velocidad y dirección del Viento mediante Redes Neuronales Artificiales. Congreso de Ingenieria de Organización Leganés, 905-914.

Pascual, Á., Subias, Á., Martín, M. L., Valero, F., García, A., Sebastian, L. I., . . Morata, A. (2010). Predicción de Velocidad Media y Rachas Máximas diarias de viento en la península lbérica mediante metodología de análogos. Asociación Española de Climatología, 571-580.

Piazzi, G. T. (2017). Snow multivariable data assimilation for hydrological predictions in Alpine sites. In EGU General Assembly Conference Abstracts, 7777.

Prieto Emhart, M. (2018). Exploring Machine Learning Models for Wind Speed Prediction. Catalunya: Universitat Politecnica de Catalunya.

Sierra-Vargas, F. E., \& Arango-Gómez, J. E. (2016). Assessment, Forecasting and modeling of wind potential. Wind Energy, 1-10.

Sun, Y. D. (2017). Optimal day-ahead wind-thermal unit commitment considering statistical and predicted features of wind speeds. . Energy Conversion and Management, 347-356.

Torrijos Moreno, J. (2019). Predicción de energía eólica con modelos autorregresivos (Bachelor’s thesis). 